\title{
Tiempos, objetos y sucesos en la metafísica estoica
}

\author{
RICARDO SALLES \\ Instituto de Investigaciones Filosóficas \\ Universidad Nacional Autónoma de México \\ rsalles@servidor.unam.mx
}

\begin{abstract}
Resumen: El propósito de este trabajo es estudiar las tesis que sostuvieron los primeros estoicos sobre la individualización de los tiempos (¿qué determina que un instante sea distinto de otro?). En contra de una objeción que formuló Jonathan Barnes contra la coherencia de la teoría estoica del eterno retorno —objeción que presupone que, para los estoicos, $t$ y $t^{*}$ son distintos sólo si los cuerpos y sucesos que se dan en $t$ son distintos de los cuerpos y sucesos que se dan en $t^{*}$-, intento mostrar que en la metafísica estoica las diferenciaciones temporales no presuponen diferenciaciones en lo que existe en el tiempo, lo cual implica que en ella puede haber tiempo sin cambio.
\end{abstract}

Palabras clave: estoicismo, sucesos tipo y sucesos caso, cualidades, disposiciones, ambigüedad

En un trabajo importante del año 1978, Jonathan Barnes intentó mostrar que la metafísica estoica encierra una contradicción. ${ }^{1}$ La supuesta contradicción surge entre la versión ortodoxa de la doctrina del eterno retorno, por una parte, y la concepción estoica del tiempo como entidad incorpórea ( $\dot{\sigma} \sigma \dot{\omega} \mu \alpha \tau o \nu \tau \iota)$, por otra. El argumento sobre el cual se apoya la objeción de Barnes puede resumirse como sigue. Según la versión ortodoxa, (A) el mundo que ahora existe habrá de repetirse cíclicamente en el futuro, sin variación alguna, un número infinito de veces; asimismo, no es otra cosa sino la repetición exacta de uno que existió infinitas veces en ciclos anteriores. Un corolario de (A) es (B): existe una diferenciación temporal entre ciclos: para cada ciclo $c_{x}$, existe un ciclo $c_{x-1}$ temporalmente anterior

\footnotetext{
${ }^{1}$ Véase Barnes 1978. Cfr. Long 1985, quien acepta la base de la objeción (a la cual llamaré más adelante la "tesis de la individualización"), pero intenta refutar la objeción recurriendo a la idea del tiempo circular y atribuyéndola a los estoicos ortodoxos. Véase también Sorabji 1983, p. 80, donde él acepta la idea básica que Barnes atribuye a los estoicos, a saber, que una diferenciación de tiempos presupone una diferenciación en los sucesos y objetos que los caracterizan: "if any time $A$ has all the same characteristics as any other time $B$, then there are no distinct times" ["si un instante $A$ cualquiera tiene todas las mismas características que otro instante $B$ cualquiera, entonces no hay dos instantes distintos"]. Como señala Sorabji, esta idea, de ser correcta, implica que el flujo del tiempo presupone el cambio: "That in turn means that, if there are to be distinct times, some change must occur, so that times $A$ and $B$ may be characterized by different states of affairs" ["Eso, a su vez, significa que si ha de haber instantes distintos, tiene que ocurrir algún cambio, de modo que los instantes $A$ y $B$ se puedan caracterizar por diferentes estados de cosas"].
} 
a $c_{x}$ y un ciclo $c_{x+1}$ temporalmente posterior a $c_{x}$. Sin embargo, Barnes afirma que (B) choca con la concepción estoica del tiempo como una entidad incorpórea, pues, según Barnes, esta concepción implica (C): aquello que individualiza a un tiempo de otro son las cualidades y disposiciones de los cuerpos que lo caracterizan; en otras palabras, para cualesquiera tiempos $t_{1}$ y $t_{2}, t_{1}$ y $t_{2}$ son dos tiempos, o instantes, distintos sólo si los cuerpos que caracterizan a $t_{1} \mathrm{y}$ los cuerpos que caracterizan a $t_{2}$ difieren en sus cualidades y disposiciones; de modo que, si no hay diferencia alguna entre lo que existe y sucede en cada uno, $t_{1}$ y $t_{2}$ no son realmente dos tiempos, sino númericamente el mismo tiempo. Por lo tanto, y ésta es la objeción, si la tesis (C) es verdadera, (A) y (B) no pueden ser ambas verdaderas. Los tiempos de cada ciclo no podrían ser distintos porque todos se caracterizarían por los mismos cuerpos con las mismas cualidades y disposiciones, lo cual, según (C) y en contra de (B), implicaría que los tiempos son, de hecho, uno solo. Para conservar la tesis (C), los estoicos deben abandonar o bien la tesis (A), que afirma que de un ciclo al otro se repite exactamente el mismo mundo, o bien su corolario (B), que afirma que los ciclos suceden en tiempos distintos. Es por esta razón, según Barnes, que los estoicos ortodoxos, quienes sostuvieron las tres tesis al mismo tiempo, entran en contradicción. ${ }^{2}$

En este trabajo me propongo defender a los estoicos ortodoxos de la objeción de Barnes. Para ello, intentaré mostrar que su concepción del tiempo como una entidad incorpórea no los obliga a aceptar la tesis (C)

\footnotetext{
${ }^{2}$ En su crítica, Barnes también recurre a una idea de la cual no me ocuparé en detalle en este trabajo, a saber, que para los estoicos los instantes del tiempo son entidades relativas; esto implica que, por no tener una existencia absoluta, dependen de otras cosas para individualizarse, a saber, de los cuerpos y sucesos que se dan en ellos. Cfr. Barnes 1978, pp. 12 y 451. Como señala el propio Barnes, esta objeción tiene antecedentes antiguos en la escuela peripatética. Véase Eudemo ap. Simplicio in Ar. phys. 732, 26-733, 1a. ed. Diels y [Aristóteles], Problemata 17.3. En la versión de Eudemo, la objeción va dirigida contra los pitagóricos (aunque también valdría para los estoicos). Tengo mis dudas, sin embargo, respecto de la pertinencia de esta crítica. En efecto, la noción de relativo usada por Barnes (y Eudemo) puede entenderse de dos maneras: en un sentido lógico (cada instante se define por los cuerpos y sucesos particulares que se dan en él) y en un sentido ontológico (los instantes dependen para su existencia de la existencia de los cuerpos que existen en ellos). Respecto del primer sentido, no hay pruebas claras de que para los estoicos ortodoxos los tiempos (y los incorpóreos en general) sean relativos en ese sentido. El argumento que desarrollo en este trabajo muestra justamente que, según los estoicos ortodoxos, la diferenciación de tiempos es independiente de la diferenciación de los cuerpos o sucesos que se dan en el tiempo: la diferenciación de tiempos - el que un instante $t$ sea anterior a $t^{*}$ y posterior a $t^{* *}$ - es un dato primitivo de la realidad. Esto implica que ningún instante se define por los cuerpos y sucesos que se dan en él. Respecto del segundo sentido, también veo problemas. No sólo es cuestionable, también en este caso, que los incorpóreos estoicos sean relativos en ese sentido (cfr. Boeri 2001): aun cuando lo fueran, de esto no se seguiría que tienen que individualizarse en función de los cuerpos de los cuales dependen, pues varios incorpóreos del mismo tipo podrían depender de un mismo cuerpo para existir. En pocas palabras, no veo que la relación de dependencia ontológica deba implicar necesariamente una relación de invidualización.
} 
que Barnes les imputa. Su objeción parte de un malentendido sobre la naturaleza de los incorpóreos estoicos. En el primer apartado ofrezco un análisis de los presupuestos de la objeción. En el segundo me ocupo de la teoría estoica de los incorpóreos. Como veremos, no hay en ella elementos que permitan concluir que para los estoicos los tiempos se individualizan por los cuerpos que los caracterizan. Ofreceré un ejemplo donde puede apreciarse claramente que los estoicos nunca tuvieron esa concepción. Este ejemplo nos llevará, en el tercer apartado, a reflexionar sobre el problema de la individualización de sucesos. Me haré la pregunta de si es coherente afirmar que los mismos sucesos ocurren en tiempos distintos (como supone la teoría ortodoxa) y, también, de cómo se aplica a sucesos el principio de la identidad de los indiscernibles.

\section{La objeción de Barnes}

La objeción de Barnes descansa sobre un supuesto general del cual la tesis (C) es un caso particular. A este supuesto lo llamaré la "tesis de la individualización" y se enuncia como sigue: si bien una misma entidad corpórea $C$ puede caracterizar a un gran número de entidades incorpóreas particulares del mismo tipo, la diferenciación de estas entidades presupone necesariamente una diferenciación en las cualidades y de las disposiciones de $C$. Según esta tesis, existe un vínculo entre caracterización e individualización: la relación de caracterización siempre determina la individualización del incorpóreo respecto de otros incorpóreos del mismo tipo. Por lo tanto, un mismo cuerpo no puede nunca caracterizar a más de un incorpóreo del mismo tipo en virtud de las mismas cualidades y disposiciones. En su artículo, Barnes no formula de modo explícito la tesis de la individualización; sin embargo, es claramente un presupuesto de su argumento. Según el autor, la razón por la cual el mismo mundo no puede caracterizar tiempos distintos es que el tiempo es un incorpóreo estoico. Esta inferencia, en efecto, no sería válida si no se supusiera la tesis de la invidualización.

Coincido con Barnes en que las entidades que los estoicos postulan como incorpóreas se caracterizan por cuerpos. Sin duda, tal es el caso de tiempo. Para tomar un ejemplo, la descripción completa de un tiempo particular como el 5 de mayo de 1862, debe hacer referencia al hecho de que es el día en que tuvo lugar la Batalla de Puebla. En esa medida, la Batalla de Puebla es una entidad corpórea característica de ese tiempo particular. Pero difiero de Barnes en cuanto a la tesis de la individualización. Para seguir con el ejemplo anterior, imaginemos que la Batalla de Puebla se hubiera repetido el 6 mayo exactamente del mismo modo y que el estado del resto del universo también se hubiese repetido. Según la tesis de la individualización, se seguiría de esto que el 5 y el 6 no serían de hecho dos tiempos distintos. Serían numéricamente el mismo tiempo con dos nom- 
bres distintos. Lo mismo ocurriría con la doctrina del eterno retorno de los estoicos ortodoxos: dado que el mundo que se repite de un ciclo a otro es exactamente el mismo, los ciclos no suceden en tiempos distintos, sino todos al mismo tiempo. En esto consiste justamente la objeción de Barnes, la cual me parece equivocada porque, según pienso mostrarlo, los estoicos nunca aceptaron la tesis de la individualización. Para ellos, la relación de caracterización entre una entidad corpórea y un incorpóreo no determina la individualización del incorpóreo. Por ello no caen en ninguna contradicción al afirmar que el mismo mundo se repite en ciclos temporalmente distintos. Antes de pasar a esto, es preciso hacer una aclaración terminológica.

En su formulación de la objeción, Barnes emplea el término "suceso": los tiempos se distinguen por los sucesos que los caracterizan. No se hace referencia a cuerpos:

El estatus ontológico de estos $\alpha \dot{\sigma} \omega \dot{\omega} \mu \alpha \tau \alpha$ no es del todo claro, pero cabe hacer la siguiente aclaración. Siendo el tiempo una entidad incorpórea, cada instante se individualiza únicamente por los sucesos que lo caracterizan: un instante no es nada sino el tiempo de los sucesos mismos; es algo relativo que carece de existencia en sí mismo, que no tiene existencia absoluta. Por lo tanto, dos instantes serán distintos si y sólo si los sucesos que caracterizan al uno se distinguen de alguna manera de los sucesos que caracterizan al otro. ${ }^{3}$

La noción de suceso, sin embargo, no afecta lo que dije antes. Pues los estoicos reducen a estados corpóreos de cosas lo que se entiende por sucesos en teorías modernas como la de Davidson. ${ }^{4}$ Pensemos en un suceso tipo como el caminar de Platón de la Academia hacia las murallas de Atenas. Según los estoicos, este suceso tipo consiste en el estado de cosas formado por Platón dispuesto de cierto modo: es Platón $\pi \hat{\omega} \varsigma$ है $\chi o v .{ }^{5}$ Más exactamente, el suceso consiste en que Platón satisfaga el predicado ( $\chi \alpha \tau \eta \gamma o ́ p \eta \mu \alpha)$ caminar

${ }^{3}$ Barnes 1978, p. 12. Cfr. p. 451: "The Stoic conception of time as incorporeal, a conception which implies that two instants of time can be distinguished only if the events which are situated in the one are distinguishable from the events which are situated in the other, would reduce the theory of an eternal recurrence, in the orthodox sense, to an absurdity, for it would reduce to a single world the worlds supposedly multiple and indefinite in their succession" [La concepción estoica del tiempo como incorpóreo, una concepción que implica que dos instantes se pueden distinguir sólo si los sucesos que están situados en uno se pueden distinguir de los sucesos situados en el otro, reduciría la teoría del eterno retorno, en el sentido ortodoxo, a un absurdo, pues reduciría a un solo mundo los mundos supuestamente múltiples e indefinidos en su sucesión"].

${ }^{4}$ Para la postura de Davidson, véanse 1967 y 1969, así como, por ejemplo, Kim y Sosa 1995, pp. 140-143.

${ }^{5}$ Véase Simplicio, in Ar. cat. Kalbfleisch, 66, 32-67, 2 (SVF 2.369; LS 27F); Plutarco, comm. not. 1083E (LS 28A); Plotino, 6.1.25.1-3 (SVF 2, 371). 
de la Academia hacia las murallas de Atenas, el cual Platón satisface en virtud de que está dispuesto de un modo específico: el de caminar de la Academia a las murallas de Atenas. La identidad del suceso tipo depende, por lo tanto, de dos elementos: (i) las cualidades en virtud de las cuales el cuerpo que camina es Platón y no otra persona, y (ii) la disposición, o

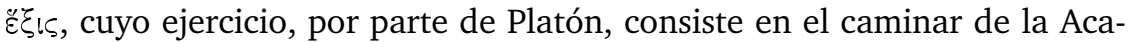
demia a las murallas de Atenas. ${ }^{6}$ Cada vez que Platón camina de un lugar al otro, ocurre un caso distinto del mismo suceso tipo. Los sucesos caso de un mismo tipo se distinguen entre sí por el tiempo en que ocurren. De esta manera, la identidad de un suceso caso se fija por la combinación de los elementos (i) y (ii) junto con un tiempo particular. Por ejemplo, el caminar de Platón de la Academia a las murallas de Atenas en $t$-el hecho de estar así dispuesto en ese momento- es un caso del suceso tipo cuya identidad es fijada por (i) y (ii).

La disposición que se menciona en (ii) puede ejercerse por un cuerpo cualificado de manera distinta. De ser así, ocurre un suceso de otro tipo; por ejemplo, el caminar de Aristóteles de la Academia a las murallas de Atenas. Así, también, un cuerpo cualificado del mismo modo que en (i) puede estar dispuesto de otra manera, en cuyo caso lo que ocurre es de nueva cuenta un suceso de otro tipo; por ejemplo, el caminar de Platón desde las murallas de Atenas de regreso a la Academia. Pero por distintos que estos sucesos sean, todos ellos consisten en el hecho, o estado de cosas, formado por un cuerpo cualificado de cierto modo y dispuesto de cierta manera. ${ }^{7}$

Ahora bien, y para regresar a la objeción de Barnes, supongamos que en el tiempo $t$ y en el tiempo $t^{\prime}$ suceden exactamente los mismos sucesos. Para simplificar, podemos suponer que el mundo sólo comprende dos sucesos caso de un mismo tipo; uno ocurre en $t$ y el otro en $t^{\prime}$ : en $t$, Platón contrae y vuelve a extender su dedo meñique, y en $t^{\prime}$, Platón vuelve a

${ }^{6}$ Para la diferencia en la ontología estoica entre cualidad y disposición, cfr. Simplicio, in Ar. cat. 212.12-213.7, Kalbfleisch, con comentario de Menn en 1999, pp. 217-227. El hecho de que una manzana sea roja puede explicarse por la presencia en la manzana de la "rojeidad", la cual es una cualidad; pero el hecho de que Platón camine no puede explicarse por la presencia en Platón de alguna cualidad, pues no hay un equivalente de "rojeidad" para caminar. Por lo tanto, para explicar el hecho de que Platón camine debe recurrirse a algo que no sea una cualidad; ésta es precisamente la función que los estoicos le atribuyen a las disposiciones: Platón camina no en virtud de una cualidad que posea, sino en virtud de estar dispuesto de cierto modo.

${ }^{7}$ En lo sucesivo, reservo los términos "hecho", "estado de cosas" y "suceso" para referirme a entidades como el caminar de Sócrates (las cuales son corpóreas, según los estoicos), y el término "proposición" para referirme a entidades como Sócrates camina, a las cuales los estoicos califican de $\dot{\alpha} \xi\llcorner\omega \mu \alpha \tau \alpha$ y $\pi \rho \alpha ́ \gamma \mu \alpha \tau \alpha$ y clasifican como incorpóreas. Respecto de las primeras, los estoicos argumentan que todo suceso es reducible a un hecho, el cual consiste en un estado de cosas, a saber, la posesión por parte de un objeto de ciertas cualidades y disposiciones. El ejemplo sobre Platón y Aristóteles lo muestra claramente. 
contraerlo y a extenderlo. Eso es lo único que ocurre en el universo. La pregunta que se haría Barnes en este caso es la siguiente: si esto fuera así, ¿podrían los estoicos afirmar que $t$ y $t^{\prime}$ son tiempos distintos? Según él, la respuesta que deberían dar es negativa: el tiempo es un incorpóreo, y una diferenciación entre incorpóreos del mismo tipo presupone una diferenciación en el cuerpo que los caracteriza; dado que en este caso no hay tal diferenciación (puesto que en $t$ y en $t^{\prime}$ el mismo cuerpo está dispuesto exactamente del mismo modo), $t$ y $t^{\prime}$ no pueden ser distintos. Como ahora veremos, sin embargo, los estoicos sí permiten la diferenciación entre incorpóreos del mismo tipo sin que haya una diferenciación en el cuerpo que los caracteriza. En otras palabras, rechazan la tesis de la individualización que Barnes les imputa. Con el fin de establecer esto, empezaré con algunas observaciones generales acerca del concepto estoico de incorpóreo.

\section{La naturaleza de los incorpóreos estoicos y su individualización}

Los estoicos eran materialistas y sostuvieron que las entidades corpóreas son las únicas dotadas de existencia sustancial. Sin embargo, reconocieron que hay ciertas cosas fundamentales para dar cuenta del mundo que, por diversas razones, no pueden ser corpóreas. Son estas cosas las que recibieron el nombre de incorpóreos $(\dot{\alpha} \sigma \dot{\omega} \mu \alpha \tau \alpha){ }^{8}$ La lista canónica de incorpóreos estoicos comprende, además del tiempo, otras tres entidades: el lugar, el vacío y los llamados "decibles", los cuales abarcan las proposiciones ( $\left.\alpha^{\prime} \xi \omega^{\prime} \mu \alpha \tau \alpha\right)$ y los predicados ( $\left.\chi \alpha \tau \eta \gamma o p \eta \dot{\mu} \mu \alpha \tau\right)$, entre otras cosas. ${ }^{9}$ No me ocuparé aquí de las razones que los estoicos tienen para afirmar que estas cuatro cosas son fundamentales para dar cuenta del mundo físico. Tampoco revisaré todos los argumentos estoicos a favor de su incorporeidad. ${ }^{10}$ En lo que sigue sólo me centraré en tres de ellos. Los primeros dos se refieren a los decibles, y el tercero, al tiempo.

Hay dos razones para pensar que los decibles no pueden ser cuerpos. La primera se relaciona con el problema de la falsedad y del significado de los enunciados falsos. Según observan Long y Sedley: "Si Catón no está caminando, el enunciado falso de que está caminando no puede tener una entidad corpórea —el Catón no caminante- como su significado." ${ }^{11}$ En otras palabras, la entidad que constituye el significado de un enunciado

\footnotetext{
8 Nótese que los incorpóreos estoicos no son las únicas entidades carentes de cuerpo que los estoicos reconocen en su ontología. Los universales estoicos carecen de cuerpo (no son entidades corpóreas), pero no por ello son $\alpha \dot{\sigma} \omega \dot{\mu} \mu \tau \alpha$. Al respecto, véanse Boeri 2001 y Salles, inédito, cap. 2.

${ }^{9}$ Para la lista canónica, véase Sexto Empírico, adv. math. 10.218 (SVF 2.331; LS 27D) y Plutarco, col. 1116B-C. Al respecto, véase Boeri 2001.

${ }^{10}$ Para un tratamiento bastante completo de este tema particular, véase Brunschwig 1994, pp. $115-126$.

11 Véase Long y Sedley 1987, vol. 1, p. 199.
} 
falso no puede ser corpórea a la manera en que lo es el significado de un enunciado verdadero. Esto hace que al menos el significado de los enunciados falsos no pueda ser cuerpos. Pero hay una razón más general por la cual ningún decible estoico puede ser corpóreo. Pensemos en el caso particular

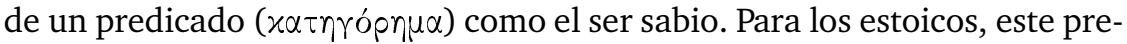
dicado no debe confundirse con Sócrates, quien es el sujeto corpóreo que lo satisface. Tampoco debe confundirse con la sabiduría, que es una cualidad corpórea, ni incluso con la expresión "es sabio", la cual es proferida por el hablante para expresar el predicado, y es también una entidad corpórea. Según los estoicos, el predicado ser sabio debe ser otra cosa, aparte de estas tres entidades corpóreas. El predicado no puede ser Sócrates porque es algo que es satisfecho por Sócrates; tampoco puede ser la sabiduría, por la simple razón de que es algo que vincula la sabiduría con Sócrates; y no puede ser la expresión "es sabio" porque el predicado es algo que esta expresión expresa. Al no ser ninguna de estas tres cosas, se agotan, en el terreno de los corpóreos, los candidatos plausibles para determinar qué es este predicado. Por lo tanto, el predicado no puede ser una entidad corpórea. ${ }^{12}$ Exceptuando quizá el significado de los nombres propios, el mismo argumento se aplicaría a las demás cosas que los estoicos clasifican como decibles y, en particular, a las proposiciones.

En el caso del tiempo, el argumento es distinto. La razón de su incorporeidad es que su corporeidad generaría un regreso al infinito. En efecto, supongamos que el tiempo en que existe un cuerpo $C$ fuera él mismo un cuerpo. De ser así, $C$ podría en principio interactuar físicamente con el tiempo mismo en que existe. En efecto, todo cuerpo puede en principio interactuar físicamente con otros cuerpos. Ahora bien, supongamos que $C$ de hecho interactúa con el tiempo $t$ en que existe. Esta interacción, como sucede con toda interacción física, tendría que suceder en algún momento $t^{\prime}$. Sin embargo, $t^{\prime}$ tiene que ser distinto de $t$ porque, ex hypothesi, $t$ es una de las dos cosas que están interactuando. Pero si $t^{\prime}$ es distinto de $t$ y también es un cuerpo, $t^{\prime}$ podría a su vez interactuar tanto con $t$ como con $C$. Pero si $t^{\prime}$ de hecho interactúa con ellos, se necesita postular otros instantes para fijar el momento en que suceden estas interacciones y, por ende, otros cuerpos. De ahí el regreso al infinito. Para que se dé algo tan simple como la existencia de algo en un momento dado, se necesita una infinidad de cuerpos, lo cual es absurdo y ontológicamente inaceptable. Por consiguiente, el tiempo no puede ser una entidad corpórea.

Ahora bien, no hay nada en ninguno de estos argumentos que pudiera sugerir que la individualización de incorpóreos del mismo tipo dependa de las cualidades y las disposiciones de los cuerpos que los caracterizan.

\footnotetext{
12 Sobre este asunto, véase Long y Sedley 1987, vol. 1, pp. 199-202, y Frede 1994. El texto fundamental es Séneca, ep. 117.13 (LS 33E). Véase, también, Sexto Empírico, adv. math. 8.11-12.
} 
La tesis de la individualización que Barnes atribuye a los estoicos es una tesis que, de hecho, nunca sostuvieron. Ofreceré dos contraejemplos para la tesis. Ambos se refieren a decibles y uno de ellos proviene de los propios estoicos.

Empiezo con un mal contraejemplo. Un diagnóstico de por qué es malo nos permitirá determinar qué debe ser un buen contraejemplo.

Imaginemos a Sócrates caminando y cantando al mismo tiempo. El modo en que está dispuesto lo hace realizar simultáneamente ambas cosas. En virtud de estar dispuesto de ese modo, Sócrates satisface simultáneamente tanto el predicado estar caminando como el predicado estar cantando. Por lo tanto, Sócrates caracteriza en ese momento ambos predicados a la vez. Sin embargo, se trata de predicados distintos. Para empezar, no son lógicamente equivalentes: el uno no implica al otro en virtud de su forma lógica. En segundo lugar, tampoco son materialmente equivalentes: hay casos posibles, distintos de éste, en que el uno es satisfecho sin que el otro lo sea. Por lo tanto, esto ejemplifica un caso en el cual un mismo cuerpo caracteriza a más de un incorpóreo del mismo tipo en virtud de una misma disposición.

Éste es un mal contraejemplo. Sin duda, puede decirse que Sócrates satisface ambos predicados en virtud de la misma disposición. Pero esta disposición es de hecho un conjunto de dos disposiciones distintas e independientes. En efecto, no atañen a las mismas partes del cuerpo de Sócrates. La primera atañe a sus piernas y todos los músculos y nervios relacionados con el movimiento de las piernas. La segunda, en cambio, atañe a sus órganos del habla y los músculos y nervios correspondientes. Las disposiciones pueden verse como una sola porque están perfectamente coordinadas la una con la otra en el cuerpo de Sócrates. Pero son disposiciones de distintas partes de su cuerpo. Por lo tanto, es falso que el cuerpo de Sócrates esté caracterizando dos predicados en virtud de una sola y misma disposición.

Un buen contraejemplo a la tesis de la individualización debe mostrar cómo un mismo cuerpo puede caracterizar a más de un incorpóreo del mismo tipo; pero, además, la disposición en cuestión no debe ser analizable en términos de disposiciones de distintas partes de ese cuerpo. Ahora bien, los estoicos piensan que esto es posible. Más aún, ofrecen un caso concreto

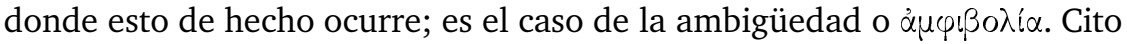
a continuación el testimonio de Diógenes Laercio:

La ambigüedad es una expresión que significa lingüísticamente y de modo estricto dos o más proposiciones de acuerdo con el mismo uso, de modo que, de acuerdo con esta expresión, las diversas 〈proposiciones〉 se comprenden.

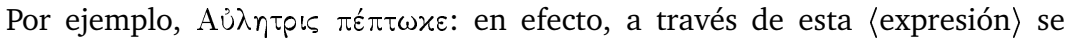

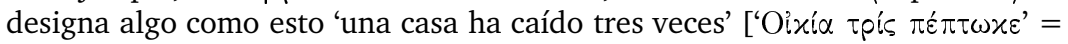




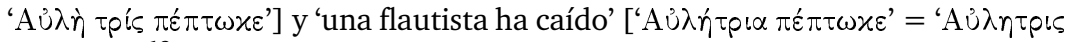
$\pi \varepsilon ́ \pi \tau \omega \varkappa \varepsilon '] .{ }^{13}$

No es preciso detenermos en cada uno de los detalles del texto. Es manifiesto que describe un caso en el cual dos o más incorpóreos del mismo tipo se caracterizan por un mismo cuerpo. Los incorpóreos en cuestión son proposiciones ( $\pi p \alpha ́ \gamma \mu \alpha \tau \alpha$ ), las cuales son entidades incorpóreas. El cuerpo, a su vez, es una "lexis" ( $\left.\lambda \varepsilon^{\xi} \xi ı\right)$ o expresión. Se trata de una entidad lingüística que consiste en una cadena articulada de sonidos proferida por un hablante. La noción de caracterización de Barnes es relevante para esta discusión. En efecto, los estoicos defendieron una teoría del significado basada en la etimología. Según esta teoría, los sonidos articulados que se emplean en el uso significativo del lenguaje guardan una relación intrínseca con sus significados. El significado de los sonidos significativos no se fija por convención, sino por la naturaleza misma del sonido. A éste se lo considera apropiado por naturaleza para expresar ese significado. El hecho de ser expresable por cierto sonido y no por otro es una característica de cada significado. ${ }^{14}$ Lo que pide la teoría, por lo tanto, es que no haya dos sonidos igualmente aptos para expresar un mismo significado. Sin embargo, esto no contradice la idea de que un mismo sonido sea la expresión natural de más de un significado. Esto es justamente lo que sucede con las expresiones ambiguas, pues significan simultáneamente dos o más proposiciones. Es importante notar que los estoicos no ofrecen una explicación pragmática de la ambigüedad. El portador de ambigüedad no es la expresión en un determinado contexto. ${ }^{15}$ Que una expresión ambigua lo sea no se debe a que haya contextos en los cuales entendemos más de una cosa; más bien hay contextos en los cuales entendemos más de una cosa porque la expresión que escuchamos es naturalmente ambigua. Hay otra razón por la cual la ambigüedad estoica no depende del contexto: es independiente de las intenciones del hablante. Según los estoicos, uno puede proferir una expresión con la intención de referirse sólo a una proposición y, a pesar de ello, significar más de una. ${ }^{16}$

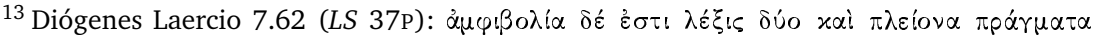

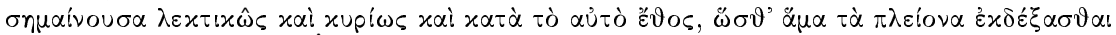

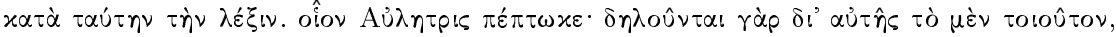

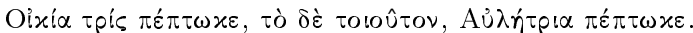

${ }^{14}$ Véase estoicos ap. Orígenes en SVF 2.146: "los sonidos primarios imitan los estados

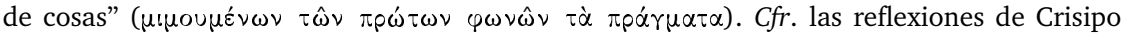

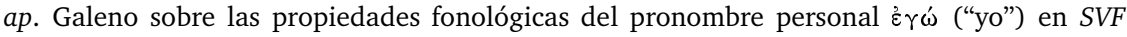
2.884. Sobre este tema, véase Lloyd 1971, Long 1986, pp. 131-139 y Atherton 1993, p. 97.

${ }^{15}$ Como lo demuestra Atherton en 1993, p. 171.

${ }^{16}$ Véase la polémica entre Crisipo y Diódoro Crono en Gelio, Noctes Atticæ 11.12.1-3 (SVF 2.152, LS 37N).
} 
Para concluir este apartado, vayamos un poco más a fondo en la noción estoica de lexis. Es evidente que para los estoicos una lexis ambigua, cuando está dotada de estructura proposicional, como es el caso en el ejemplo de Diógenes, caracteriza más de una proposición en virtud de una misma disposición. En efecto, la lexis no es otra cosa que cierto volumen de aire dispuesto de manera que suene de cierto modo. En el nivel más básico, es este volumen de aire lo que caracteriza, en virtud de esa disposición, las dos proposiciones incorpóreas que menciona el texto. También es evidente que esta disposición no es, a su vez, analizable en términos de disposiciones de distintas partes de la lexis. La idea no es que cada una de las proposiciones sea significada por partes distintas de la lexis. La idea es, más bien, que la lexis significa ambas proposiciones en virtud de una sola de sus partes, que es la misma en ambos casos. Esta parte es la cadena de sonidos $\langle\alpha \dot{v}, \lambda \eta$, тpıs〉, en la cual radica la ambigüedad de la lexis.

Así, pues, pretendo haber establecido que los estoicos no aceptan la tesis de la individualización que Barnes les atribuye. Para ellos es perfectamente posible que un mismo cuerpo caracterice más de un incorpóreo del mismo tipo en virtud de una misma disposición. Por lo tanto, la concepción estoica del tiempo como incorpóreo es compatible con la idea de que dos tiempos distintos se caractericen por un mismo conjunto de cualidades y disposiciones de un mismo conjunto de cuerpos. Esto refuta la objeción de Barnes.

\section{Tiempos e individualización de sucesos}

En lo que sigue deseo ocuparme de un tema distinto, pero complementario, del que acabo de discutir. Vimos que la concepción estoica del tiempo no va en contra de la idea de que el mismo mundo se repite en tiempos distintos. En este apartado quiero preguntarme si acaso hay algo en la concepción estoica de suceso que vaya en contra de esa idea. Lo que está en juego es, una vez más, la coherencia de la versión ortodoxa de la teoría del eterno retorno. Si exactamente el mismo mundo se repite en tiempos distintos, los sucesos que ocurren en él también deben repetirse y ser los mismos en un tiempo y en el otro. Pero ¿puede un mismo suceso ocurrir en tiempos distintos?

La pregunta de si un mismo suceso puede ocurrir en tiempos distintos de hecho admite tanto una respuesta negativa como una positiva. Cuál de ellas sea correcta depende de cómo entendamos el tipo de identidad que atribuimos al suceso.

Si cuando decimos "el mismo suceso" nos referimos al mismo suceso caso, la respuesta a la pregunta debe ser negativa. En efecto, un suceso caso se individualiza por el momento en que ocurre. ${ }^{17}$ Por lo tanto, si el tipo de identidad que los estoicos tienen en mente al afirmar que los

\footnotetext{
${ }^{17}$ A diferencia de los objetos, los cuales no se individualizan por el tiempo en que existen: un objeto que persiste de $t$ a $t^{\prime}$ no debe analizarse, según los estoicos, en términos de la suma
} 
mismos sucesos ocurren en un ciclo y en el otro, es una identidad caso, se sigue que sería contradictorio de su parte sostener que el mundo actual es temporalmente anterior al mundo del próximo ciclo.

Sin embargo, si cuando decimos "el mismo suceso" entendemos el mismo tipo de suceso, la respuesta a la pregunta anterior puede ser afirmativa. Esto se debe, a su vez, al hecho de que puede haber una identidad tipo entre sucesos que ocurren en tiempos distintos. En este caso no hay ninguna contradicción al afirmar que los mismos sucesos se repiten de un ciclo al otro. Lo que esto significa es simplemente que el tipo de sucesos que ocurren en el mundo actual es idéntico al tipo de sucesos que ocurren en el mundo del próximo ciclo. Esta identidad puede ser estricta y, en todo caso, debe serlo para que tenga sentido la idea estoica de que no hay variación alguna de un ciclo al otro. Por ejemplo, Ricardo fue al cine en la tarde del 10 de septiembre de 2001 de este ciclo. Así también, Ricardo irá al cine en la tarde del 10 de septiembre de 2001 del próximo ciclo. El tipo de cosa que hace Ricardo - el suceso tipo- es ir al cine por la tarde el 10 de septiembre de 2001. Un ejemplo caso de ese tipo ocurre en este ciclo, y otra en el próximo ciclo. De hecho, el tipo de suceso tiene infinitas instancias caso, pues, según los estoicos, el número de ciclos es infinito. Regresaré a esta idea un poco más adelante; pero la conclusión que cabe extraer es que los mismos sucesos pueden ocurrir en un ciclo y en otro si la identidad en cuestión se da entre tipos de sucesos, y no entre sucesos caso.

Lo que necesitamos saber, por lo tanto, es si la repetición cíclica de sucesos que postulan los estoicos sólo involucra una identidad tipo de sucesos o también una identidad caso. Para determinar esto es preciso acudir a los textos. Me parece que los estoicos tenían en mente una identidad tipo; por ello, considero que no caen en ninguna dificultad lógica al afirmar una repetición cíclica de sucesos. Sin embargo, no todos los estudiosos del estoicismo comparten esta opinión. Un caso notable es el de Long y Sedley. Según ellos, la identidad transcíclica que afirman los estoicos es una identidad que implica indiscernibilidad, la cual, a su vez, implica identidad numérica. ${ }^{18}$ Pero la identidad numérica entre el mundo del ciclo presente y el mundo del ciclo siguiente pide que los sucesos que ocurren en este ciclo sean numéricamente los mismos que los sucesos del ciclo siguiente. En efecto, el mundo no podría ser numéricamente el mismo de un ciclo al

de dos objetos, uno que existe en $t$ y otro que existe en $t^{\prime}$, pues un mismo objeto puede existir en dos instantes distintos. Esto vale aun cuando el objeto sufra cambios de $t$ a $t^{\prime}$. De esto me ocupo en detalle en Salles 1999.

${ }^{18}$ En esto estoy de acuerdo con Long y Sedley. Confróntese el principio del siguiente apartado. Los estoicos afirmaron la indiscernibilidad transcíclica del mundo a la vez que aceptaron la tesis de que indiscernibilidad implica identidad numérica. Donde difiero de Long y Sedley es en (i) la tesis de que el principio de la identidad de indiscernibles se aplica a sucesos caso, y, por consiguiente, en (ii) la tesis de que los sucesos que se repiten de un ciclo al otro en la versión ortodoxa son numéricamente los mismos. 
otro, a menos que los sucesos que ocurren en él también fueran numéricamente los mismos. Por lo tanto, y ésta es la conclusión del argumento, no se repiten de un ciclo al otro meras instancias distintas del mismo tipo, sino los mismos sucesos caso. Esto conduce a los estoicos a un problema lógico, porque, como ya vimos, el mismo suceso caso no puede ocurrir en tiempos distintos. Cito a continuación las palabras de Long y Sedley:

Si $t$ es el conjunto de instantes del mundo presente y $t^{*}$ el conjunto de instantes de la próxima repetición de este mundo, los movimientos que ocurren en la dimensión temporal $t^{*}$ deben ser posteriores a $t$. Pero ex hypothesi [i.e. dada la identidad por indiscernibilidad que postulan los estoicos] los movimientos que ocurren en $t$ son numéricamente distintos de aquellos que ocurren en $t^{*}$. Por lo mismo, un movimiento que ocurre en el instante $t$, el cual es anterior a $t^{*}$, es numéricamente idéntico a un movimiento que ocurre en el instante $t^{*}$. Sin embargo, esto es incoherente de acuerdo con una concepción lineal del tiempo, pues implica que un mismo movimiento está dotado de dos dimensiones temporales diferentes. ${ }^{19}$

En lo que sigue pretendo hacer dos cosas. En primer lugar, intentaré mostrar, apoyándome en textos, que para los estoicos lo que se repite son meramente instancias distintas del mismo suceso tipo. En segundo lugar, argumentaré, apoyándome en consideraciones lógicas, que esto no viola en lo más mínimo la tesis de la indiscernibilidad.

Hay cuandos menos un texto que va en contra de la interpretación de Long y Sedley. ${ }^{20}$ Se trata de un pasaje del comentario de Alejandro de Afrodisia a los Segundos Analíticos. El texto se refiere a la concepción que tenía Crisipo de la identidad transcíclica:

Está establecido por los estoicos que, después de la conflagración, todas las cosas en el universo vuelven a ser las mismas en número porque piensan que, en el universo posterior, incluso el que está cualificado de una manera peculiar es y deviene de nuevo el mismo que el de antes, como dice Crisipo en los libros del De Mundo... ${ }^{21}$

${ }^{19}$ Long y Sedley 1987, vol. 1, p. 312: "If $t$ is the set of instants for the present world, and $t^{*}$ is the set on instants for the next recurrence of that world, $t^{*}$ must be a temporal dimension of movements all of which occur after $t$. But ex hypothesi the movements which occur at $t$ are numerically identical to those which occur at $t^{*}$. Hence, a movement at $t$, which is before $t^{*}$, is numerically identical to a movement at $t^{*}$. Now on a linear conception of time this is incoherent: for it implies two quite separate temporal dimensions for and the same movement."

${ }^{20}$ Véase también Nemesio, nat. hom. 111, 20-23 y 112, 1-3, ed. Morani.

${ }^{21}$ Alejandro de Afrodisia, in Ar. a. pr. 180, 33-6, ed. Wallies (SVF 2.624, LS 52F): ápé $\sigma x \varepsilon !$

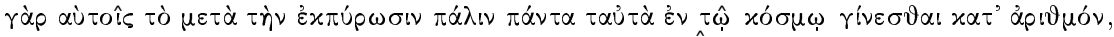

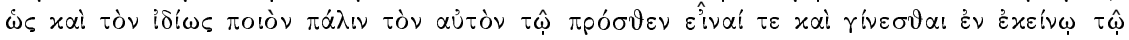

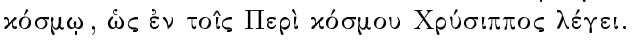


Aquí cabe hacer dos observaciones. Ambas van en contra de la interpretación de Long y Sedley. Para empezar, el griego de la primera oración es ambiguo. "Todas las cosas en el mundo vuelven a ser las mismas en núme-

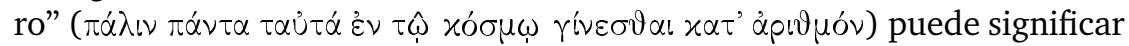
una de dos cosas: (i) numéricamente las mismas cosas se generan de un

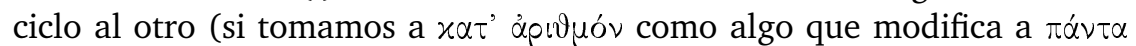
$\tau \alpha u ̛ \tau \alpha ́$ en vez de a $\gamma^{\prime}(v \varepsilon \sigma \vartheta \alpha$ ), o (ii) la generación de las mismas cosas es,

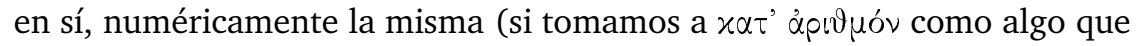

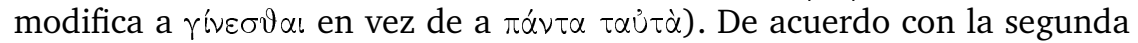
lectura, la identidad numérica atañe al suceso mismo que consiste en la generación de las cosas. Pero según la primera lectura, la identidad numérica sólo atañe a las cosas que se generan. Su generación es un suceso que ocurre un número infinito de veces, una vez en cada ciclo. Debido a que ocurre infinitas veces, no es numéricamente el mismo de un ciclo al otro. Por consiguiente, esta lectura perfectamente posible del texto deja un amplio margen para la idea de que, en lo que se refiere a sucesos, la identidad transcíclica es únicamente de tipo.

La otra observación se refiere a la segunda oración del texto: la cláusula de $\dot{\omega} \varsigma$. La primera oración, como ya vimos, alude vagamente a "cosas" sin especificar si se trata de objetos o sucesos. Pero la segunda alude inequívo-

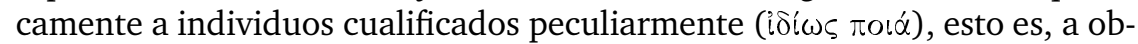
jetos. ${ }^{22}$ En efecto, según la ontología estoica son objetos, y no sucesos, las entidades que están cualificadas peculiarmente. ${ }^{23}$ Esto sugiere, retrospectivamente, que las entidades a las cuales aludía la primera oración también eran objetos. En ese caso, el argumento que Alejandro atribuye a Crisipo sería el siguiente: dado que numéricamente los mismos objetos se repiten de un ciclo al otro, los objetos del presente tienen las mismas cualidades peculiares que los objetos del siguiente. Esto es una consecuencia lógica de las nociones crisipianas de "cualidad peculiar" (iớa rotótns) y de "entidad

${ }^{22}$ Sobre la noción de cualidad peculiar y de entidad cualificada peculiarmente, véase Simplicio, in Ar. de an. 217, 36-218, 2, ed. Hayduck (SVF 2.395, pp. 130, 43-47; LS 28I), Alejandro de Afrodisia in Ar. a. pr. 181, 25-31, ed. Wallies (SVF 2.624, p.190, 3-9; LS 52F2-3); Estobeo, ecl. 1.117, 21-179, 17 (LS 28D), y Déxipo, in Ar. cat. 30, 20-6, ed. Busse (LS 28J). Para una discusión detallada de estas nociones, véase Sedley 1982. Su función es explicar la diferencia numérica entre individuos del mismo tipo o especie. Así, los objetos numéricamente distintos de una misma especie siempre están "peculiarmente cualificados", en el sentido de que cada uno posee cualidades inherentes que lo distinguen qua individuo y que, por ello, perduran a lo largo de la existencia del objeto. La tesis fuerte de la metafísica estoica a este respecto es la afirmación de que hay tales cualidades. En las fuentes antiguas no está del todo claro en qué consistían dichas cualidades.

${ }^{23}$ Podría afirmarse que un suceso estoico tiene una cualidad peculiar, pero sólo en un sentido derivado. Por ser cuerpos cualificados y dispuestos de cierto modo (cfr. apartado 1), la individualización de un suceso caso se determina, entre otras cosas, por la cualidad peculiar del cuerpo así dispuesto. Pero, en sentido estricto, el portador de la cualidad peculiar es el cuerpo y no el suceso; en otras palabras, el portador es el cuerpo que tiene la cualidad y no el hecho de que el cuerpo esté así cualificado. 


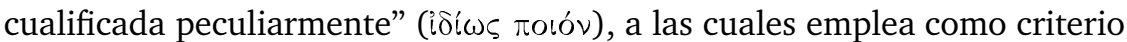
de identidad numérica: $a$ y $b$ son numéricamente el mismo objeto si, y sólo si, $a$ y $b$ son el mismo individuo cualificado peculiarmente (o poseen la misma identidad peculiar). Dicho de otro modo, para cada entidad individual existe una cualidad peculiar en virtud de la cual es numéricamente distinta de otros individuos. Por lo tanto, si interpretamos el pasaje de esta manera, aparece una conexión lógica muy estrecha entre la metafísica de Crisipo y el argumento que Alejandro le atribuye. Me parece que esto representa una razón de peso para pensar que, en este pasaje, y en contra de lo que afirman Long y Sedley, la identidad transcíclica es numérica sólo en el caso de objetos.

Antes de pasar al segundo tema del que prometí ocuparme —que la indiscernibilidad de sucesos no implica su identidad numérica-, quisiera ilustrar con un ejemplo las implicaciones que tiene afirmar que la identidad transcíclica sólo es numérica en el caso de objetos.

Sabemos que Bruto asesinó a César en el año 44. Pues bien, según la versión ortodoxa de la doctrina del eterno retorno, este suceso habrá de repetirse en el año 44 del próximo ciclo. De hecho, Bruto asesinará a César un número infinito de veces, una vez en cada ciclo. Ahora bien, como esto lo indica, el suceso que consiste en que Bruto asesine a César no es numéricamente el mismo a través de los ciclos. Pero es numéricamente el mismo Bruto quien asesinará (y ha asesinado) a César un número infinito de veces y numéricamente el mismo César quien morirá (y ha muerto) a manos de Bruto un número infinito de veces. Son numéricamente los mismos los objetos involucrados en los sucesos que se repiten de un ciclo al otro, aunque los sucesos que se repiten a lo largo de los ciclos no sean ellos numéricamente los mismos: son apenas instancias exactamente del mismo tipo. Los estoicos ortodoxos se refieren a esta identidad tipo estricta mediante la tesis de que todos los objetos que existen en el ciclo presente, así como todos los sucesos que ocurren en él, son indiscernibles ( $\alpha \dot{\pi} \alpha \rho \alpha \lambda \lambda \alpha \dot{\alpha} \chi \tau \circ$ ) de los objetos y sucesos de cualquier otro ciclo. ${ }^{24}$

\section{Indiscernibilidad de sucesos sin identidad numérica}

Vimos que no hay nada en el concepto estoico de suceso que impida lógicamente que más de un tiempo sea caracterizado por un mismo cuerpo en virtud de las mismas cualidades y disposiciones. Ese concepto de suceso es, por lo tanto, plenamente compatible con la tesis ortodoxa de que el mismo mundo se repite en tiempos distintos. Una cosa es compatible por una sen-

${ }^{24}$ Cfr. Nemesio, nat. hom. 111, 20-23 y 112, 1-3, ed. Morani, y Alejandro de Afrodisia in Ar. a. pr. 181, 25-31, ed. Wallies (SVF 2.624, p. 190, 3-9; LS 52F2-3). No me detendré aquí en las razones que llevaron a los estoicos ortodoxos a afirmar esta indiscernibilidad. Para ello, véase Mansfeld 1979 y 1981, y Salles 1999 y el inédito, cap. 1. 
cilla razón: para los estoicos, los sucesos que se repiten de un ciclo al otro (y, por lo mismo, en tiempos distintos) son casos distintos del mismo tipo. Esto es perfectamente coherente por lo que respecta al concepto estoico de suceso; en efecto, casos distintos de un mismo tipo no sólo pueden, sino que deben ocurrir en tiempos distintos.

En este último apartado intentaré refutar una posible objeción al argumento que acabo de desarrollar: ¿es coherente la idea de que los sucesos del ciclo presente y los sucesos de otro ciclo sean casos distintos del mismo tipo? La duda es pertinente: afirmar que son casos distintos implica decir que son numéricamente distintos; pero, si la identidad tipo que hay entre ellos es completa e implica indiscernibilidad, parece seguirse que, en realidad, no pueden ser numéricamente distintos. En efecto, los estoicos ortodoxos sostuvieron que la indiscernibilidad implica la identidad numérica. A esta tesis, cuya fama debemos a Leibniz pero que tiene su origen en los estoicos, se la conoce como el "principio de la identidad de indiscernibles". ${ }^{25}$ Por lo tanto, parece encerrar una contradicción la idea de que los sucesos que se repiten de un ciclo al otro son numéricamente distintos. De esta objeción me ocuparé en este apartado.

Para refutarla intentaré disipar un malentendido del que parte. Como veremos, los estoicos aceptaron el principio de la identidad de indiscernibles, pero limitaron su aplicación a objetos. Cuando se trata de sucesos caso, por ejemplo, la indiscernibilidad no implica — ni debe implicar- la identidad numérica. Esto se debe a dos razones que explicaré en detalle. Quisiera primero indicar brevemente en qué consisten. La primera razón puede calificarse de metafísica: el tiempo de un suceso caso ayuda a individualizarlo como tal, pero no por ello es una cualidad o propiedad del suceso. Por lo tanto, dado que el principio de la identidad de indiscernibles se refiere a las entidades por sus propiedades, el principio no abarca el tiempo de un suceso. Por ello puede haber sucesos caso indiscernibles que ocurren en tiempos distintos. La segunda razón es de orden sintáctico. Como veremos, la forma lógica del principio no implica de modo alguno que la indiscernibilidad de sucesos caso implique su identidad numérica.

Veamos el primer argumento. Contra la afirmación de que dos sucesos caso indiscernibles pueden ocurrir en tiempos distintos, podría objetarse lo siguiente: si los sucesos fueran realmente indiscernibles, compartirían las mismas propiedades; por lo tanto, tendrían que ocurrir al mismo tiempo; de otro modo, serían discernibles, a saber, en cuanto al tiempo en que ocurren. Esta objeción sólo es válida si se presupone que el tiempo de un suceso constituye una de sus propiedades. Pero este supuesto es cuestionable desde un punto de vista conceptual. Para mostrarlo, me apo-

${ }^{25}$ Véase Leibniz 1680. Para los estoicos, véase Cicerón acad. 2.83-5 (LS 40J). 
yaré en consideraciones kantianas. ${ }^{26} \mathrm{El}$ tiempo en que ocurre un suceso no puede considerarse como una de sus propiedades, si por "propiedad" entendemos un objeto posible de experiencia. Esto es: $F$ es una propiedad de $a$ sólo si podemos experimentar $a$ como $F$. En este sentido, ser rojo puede ser una propiedad pues podemos experimentar una entidad como roja (o incluso el hecho de que sea roja). Lo mismo sucede con propiedades como camina y ser el padre de, a las cuales los estoicos calificarían de predicados que corresponden, respectivamente, a una disposición intrínseca y a una disposición relativa. El problema con el tiempo es que no constituye un objeto posible de experiencia. Aquí hay tres problemas. En primer lugar, del hecho de que experimentemos una entidad en un tiempo determinado, no se sigue que el tiempo en cuestión sea él mismo un objeto de esa experiencia. En segundo lugar, lo primero es de hecho incompatible con lo segundo: si experimentamos una entidad en un tiempo determinado, ese tiempo no puede ser él mismo un objeto de esa experiencia. Si lo fuera, caeríamos en círculo: el tiempo sería a la vez objeto y presupuesto lógico de la misma experiencia. El tercer problema es el siguiente: aun cuando el tiempo de una experiencia pudiera ser objeto, no de ella, sino de otra experiencia (cosa que el propio Kant difícilmente aceptaría) tiene que haber al menos una experiencia cuyo tiempo no es objeto de ninguna; de lo contrario, caeríamos en un regreso al infinito. En efecto, no son posibles experiencias atemporales y, como ya vimos, una experiencia no puede tener como objeto su propio tiempo; por lo tanto, necesitaríamos otro tiempo para fechar la segunda experiencia, tiempo que sería, a su vez, objeto posible de una tercera experiencia y así hasta el infinito. Debido a estos tres problemas, el tiempo no puede ser objeto de experiencia. Por consiguiente, el tiempo no puede ser una propiedad de sucesos. ${ }^{27}$ Por esta razón puede haber sucesos indiscernibles que ocurren en tiempos distintos.

Veamos ahora el segundo argumento. Éste se refiere a la forma lógica del principio de identidad de indiscernibles. Su objetivo es mostrar que ésta permite hablar sin contradicción de sucesos caso indiscernibles pero numéricamente distintos.

Imaginemos dos sucesos caso arbitrarios $s_{a}$ y $s_{b}$. Ambos son entidades corpóreas analizables en términos de otras entidades corpóreas más básicas: objetos, por un lado, y cualidades y disposiciones, por otro. ${ }^{28}$ En

\footnotetext{
${ }^{26}$ Véase Kant 1787, A33-37/B49-54, con comentario de Stepanenko 1995, pp. 149-150, y van Cleve 1999, pp. 44-61.

${ }^{27}$ Para un argumento que llega a esta misma conclusión sin recurrir a consideraciones kantianas, cfr. Millikan 1984, pp. 261 y 283-296, esp. la 289. En la p. 261, Millikan se refiere a la teoría estoica del eterno retorno como algo que contradice el principio de la identidad de indiscernibles. Esto me parece erróneo: para sostener la teoría no es necesario abandonar el principio, sino sólo mostrar que su campo de aplicación no abarca sucesos caso. Véase el segundo argumento.

${ }^{28}$ Nótese que un objeto o cuerpo no es idéntico ni a sus cualidades ni al hecho de que las
} 
concreto, $s_{a}$ se define por la posesión de ciertas cualidades y disposiciones por parte del objeto $a$ en un tiempo $t$; $s_{b}$, en cambio, por la posesión de ciertas cualidades y disposiciones por parte del objeto $b$ en un tiempo $t^{\prime}$. Ahora bien, supongamos que los sucesos $s_{a}$ y $s_{b}$ son indiscernibles, esto es, exactamente del mismo tipo. El que sean del mismo tipo significa que los objetos que involucran tienen exactamente las mismas cualidades y disposiciones. Lo que acabo de decir puede expresarse en lenguage simbólico sin referirnos explícitamente, en ese lenguaje, a los sucesos $s_{a}$ y $s_{b}$, sino únicamente a los objetos, cualidades y disposiciones que los componen:

$$
(Q)(D)((Q a \equiv Q b) \&(D a \equiv D b))
$$

Ahora bien, los sucesos $s_{a}$ y $s_{b}$ serían numéricamente el mismo suceso sólo si la tesis (2) fuera el caso, esto es, sólo si la posesión de $Q$ y de $D$ por parte de $a$ fuera ella misma idéntica a la posesión de $Q$ y de $D$ por parte de $b:^{29}$

$$
Q a=Q b \& D a=D b .
$$

Sin embargo, de (1) —esto es, de la tesis que corresponde a la indiscernibilidad entre $s_{a}$ y $s_{b}-$ no se sigue (2), como puede verse en (3):

$$
\neg(((Q)(D)((Q a \equiv Q b) \&(D a \equiv D b))) \rightarrow(Q a=Q b \& D a=D b)) .
$$

Si algo se sigue de (1) es que los objetos $a$ y $b$ son numéricamente el mismo, como puede verse en (4):

$$
((Q)(D)((Q a \equiv Q b) \&(D a \equiv D b))) \rightarrow a=b .
$$

Pero (4) no es otra cosa sino el principio de la identidad de los indiscernibles, el cual afirma que dos objetos individuales cualesquiera son de hecho el mismo objeto numéricamente si poseen las mismas cualidades y disposiciones. El meollo del argumento está en la definición estoica de suceso, la cual afirma que todo suceso es un cuerpo dotado de ciertas cualidades y disposiciones. Si aceptamos esta definición, la indiscernibilidad, o identidad tipo, entre sucesos casos implica la identidad numérica de los objetos involucrados en los sucesos, pero no así la de los sucesos mismos.

posee (Platón no es ni la blancura ni el hecho de que posee blancura). Lo mismo se aplica a un cuerpo y sus disposiciones. Esto implica que tanto los $\pi$ oเ $\alpha$ como los $\pi \hat{\omega} \varsigma$ है $\chi$ ov $\tau \alpha$ de los estoicos son analizables en términos de entidades más básicas irreducibles las unas a las otras: el cuerpo que está cualificado y dispuesto, por un lado, y las cualidades y disposiciones que posee, por otro. Al respecto, véase Menn 1999, p. 222, n. 10.

${ }^{29}$ La identidad numérica entre $s_{a}$ y $s_{b}$ presupondría, también, la identidad de los tiempos $t$ y $t^{\prime}$ pues dos sucesos son numéricamente el mismo sólo si los tiempos en que suceden son el mismo ( $c f r$. apartado 1). Esto es, el enunciado en lenguaje simbólico que correspondería a la afirmación en metalenguaje de la identidad numérica entre $s_{a}$ y $s_{b}$ sería, en su versión más completa, la conjunción de (2) con la identidad numérica entre $t$ y $t^{\prime}$, esto es (2)': $(Q a=Q b \& D a=D b) \&\left(t=t^{\prime}\right)$. 
La formulación del principio que encontramos en los estoicos es bastante explícita a este respecto. Según ella, lo que es imposible es que haya dos objetos exactamente iguales. Esto lo apunta Cicerón, quien al referirse a los estoicos afirma que: "no hay un cabello o grano de arena que sea en todos los aspectos como (omnibus rebus talem qualis) otro cabello o grano de arena". ${ }^{30} \mathrm{El}$ latín dice talem qualis. Esto puede referirse no sólo a las cualidades de los objetos, sino también a sus disposiciones, ya sea intrínsecas o relativas. Pero independientemente de cómo interpretemos la oración, la identidad numérica se aplica a los objetos que son iguales y no a su posesión de las cualidades y/o disposiciones que hacen que sean iguales. Por lo tanto, llegamos nuevamente a la conclusión de que puede haber sucesos caso indiscernibles pero numéricamente distintos.

En resumen, si aceptamos la definición estoica de suceso, no hay buenas razones para suponer que la indiscernibilidad de sucesos caso implique su identidad numérica. La tesis ortodoxa de que los sucesos del presente ciclo son indiscernibles de los sucesos de los ciclos pasados y futuros, no constituye una violación del principio de la identidad de indiscernibles.

\section{Conclusión}

La objeción de Jonathan Barnes queda refutada: la metafísica estoica no incluye elemento alguno que contradiga la idea de que un mismo cuerpo puede caracterizar más de un tiempo en virtud de las mismas cualidades y disposiciones.

Esta idea no choca ni con el concepto estoico de incorpóreo ni con su concepto de suceso. La noción estoica de ambigüedad demuestra que un mismo cuerpo puede caracterizar a más de un incorpóreo del mismo tipo sin que a esta diferenciación corresponda una diferenciación en las cualidades y disposiciones del cuerpo. Asimismo, es perfectamente coherente la idea de que dos sucesos caso indiscernibles sucedan en tiempos distintos y, por ello, sean numéricamente distintos. En efecto, el tiempo no es una propiedad de los sucesos y el principio estoico de la identidad de indiscernibles no implica la identidad numérica cuando se trata de sucesos caso.

Si todo lo anterior es correcto, entonces, contra lo que ha sugerido Barnes, podemos interpretar literalmente la versión ortodoxa del eterno retorno sin incurrir en ninguna falla lógica. ${ }^{31}$

${ }^{30}$ Cicerón, acad. 2.83-5 (LS 40J).

${ }^{31}$ Quiero agradecer a mis colegas del Instituto de Investigaciones Filosóficas: Atocha Aliseda, Axel Barceló, Gustavo Fondevilla, Guillermo Hurtado, Claudia Lorena García, Faviola Rivera y Pedro Stepanenko sus comentarios críticos a una versión anterior de este trabajo. La investigación fue realizada en el marco de los proyectos CONACYT J30724-H, PAPIIT IN401799 y PAPIIT IN401301. 


\section{BIBLIOGRAFÍA}

Atherton, C., 1993, The Stoics on Ambiguity, Cambridge University Press, Cambridge.

Barnes, J., 1978, "La Doctrine du retour éternel", en J. Brunschwig (comp.), Les Stoïciens et leur logique, CNRs-Vrin, París, pp. 3-20.

Boeri, M., 2001, "The Stoics on Bodies and Incorporeals", Review of Metaphysics, vol. 54, pp. 723-752.

Brunschwig, J., 1994, “The Stoic Theory of the Supreme Genus and Platonic Ontology", en Papers in Hellenistic Philosophy, Cambridge University Press, Cambridge, pp. 92-157 (versión original en francés de 1988).

Cleve, J. van, 1999, Problems from Kant, Oxford University Press, Oxford.

Davidson, D., 1980, Essays on Actions and Events, Oxford University Press, Oxford [versión en castellano: Ensayos sobre acciones y sucesos, trad. Olbeth Hansberg, J.A. Robles y Margarita M. Valdés, Instituto de Investigaciones FilosóficasUNAM/Crítica, Barcelona, 1995].

—_ 1969, "The Individuation of Events", en Davidson 1980, pp. 163-180 [versión en castellano: "La individuación de los sucesos", pp. 205-229].

_ 1967, "The Logical Form of Action Sentences", en Davidson 1980, pp. 105122 [versión en castellano: "La forma lógica de las oraciones de acción", pp. 133-153].

Frede, M., 1994, "The Stoic Notion of a Lekton", en Everson (comp.), Companions to Ancient Thought 3. Language, Cambridge University Press, Cambridge, pp. 109128.

Kant, I., 1787, Critique of Pure Reason, trad. N. Kemp Smith, St. Martin's Press, Nueva York, 1965.

Kim, J., y E. Sosa (comps.), 1995, A Companion to Metaphysics, Basil Balckwell, Oxford.

Leibniz, G., 1680, "On the Principle of Indiscernibles", en M. Morris y G.H.R. Parkinson (comps.), Leibniz: Philosophical Writings, Dent, Londres, 1973.

Lloyd, A.C., 1971, "Grammar and Metaphysics in the Stoa", en A.A. Long, Problems in Stoicism, The Athlone Press, Londres, pp. 58-74.

Long, A.A., 1986, Hellenistic Philosophy, 2a. ed., Duckworth, Londres.

—, 1985, "The Stoics on World-Conflagration and Everlasting Recurrence", en R.H. Epp (comp.), Recovering the Stoics. Spindel Conference 1984, suplemento de Southern Journal of Philosophy, 23, pp. 13-37.

Long, A.A. y D.N. Sedley, 1987, The Hellenistic Philosophers, Cambridge University Press, Cambridge.

Mansfeld, J., 1981, "Bad World and Demiurge: A 'Gnostic' Motif from Parmenides and Empedocles to Lucretius and Philo", en R. van den Broeck y M.J. Vermaseren (comps.), Gnosticism and Hellenistic Religions, E.J. Brill, Leiden, pp. 261-314.

-1979, "Providence and the Destruction of the Universe in Early Stoic Thought", en M.J. Vermaseren (comp.), Studies in Hellenistic Religions, E.J. Brill, Leiden, pp. 129-188.

Menn, S., 1999, "The Stoic Theory of Categories", Oxford Studies in Ancient Philosophy, vol. XVII, pp. 215-247. 
Millikan, R.G., 1984, Language, Thought and Other Biological Categories, "Part IV: Theory of Identity", The MIT Press, Cambridge, Mass./Londres.

Salles, R., 1999, "Eterno retorno, determinismo y tiempo en la filosofía estoica", en L. Benítez y J.A. Robles (comps.), Materia, espacio y tiempo: de la filosofía natural a la física, Facultad de Filosofía y Letras-UnAM, México, pp. 183-203.

— inédito, The Stoics on Determinism and Compatibilism.

Sedley, D.N., 1982, "The Stoic Criterion of Identity", Phronesis, vol. 27, pp. 255275.

Sorabji, R., 1983, Time, Creation and the Continuum, Duckworth, Londres.

Stepanenko, P., 1995, "Conciencia y autoconciencia en Kant", Diánoia, vol. XLI, no. 41 , pp. $145-155$.

Recibido el 8 de noviembre de 2001; revisado el 21 de junio de 2002; aceptado el 25 de junio de 2002 